\title{
Blindly inserted nasogastric feeding tubes and thoracic complications in intensive care
}

\author{
Elpis Giantsou*, Kevin J. Gunning \\ John V. Farman Intensive Care Unit, Addenbrooke’s Hospital, Cambridge University Hospitals NHS Trust, England, UK; \\ *Corresponding Author: elpisgiantsou@yahoo.com
}

Received 4 July 2010; revised 13 July 2010; accepted 20 July 2010.

\begin{abstract}
Purpose of review: This article reviews the thoracic complications from malpositioned blindly inserted nasogastric feeding tubes in mechanically ventilated patients in intensive care and the methods to check the position and promote safe placement of the feeding tubes. Recent findings: Malpositioned feeding tubes are not included in risk management databases. The reported incidence is $1-3 \%$ and more than half occur in mechanically ventilated patients. Eighty three mechanically ventilated patients were reported with malpositioned nasogastric tubes and $66 \%$ of them developed serious thoracic complications. Pneumothoraces accounted for $80 \%$ of thoracic complications that were evenly distributed between tubes with and without stylet. Repeated misplacements appear to increase the risk. Nonradiological confirmation of the position of the tube has suboptimal performance. Protocols to place feeding tubes and new technology are promising candidates. Summary: Malpositioned nasogastric feeding tubes are underreported and associated with serious thoracic complications in mechanically ventilated patients. We need more data to answer whether we can afford to prevent them.
\end{abstract}

Keywords: Malpositioned Nasogastric Feeding Tube; Thoracic Complications and Mechanical Ventilation

\section{INTRODUCTION}

Critically ill patients, who cannot meet their daily nutriant requirements by oral intake but have a functional gastrointestinal tract should be considered for enteral feeding [1]. Although usually considered a simple procedure the blind placement of nasogastric feeding tubes is not without risks. This is the reason why the National Patient Safety Agency in the United Kingdom has recently issued a safety alert regarding the placement of nasogastric feeding tubes in neonatal intensive care units [2]. More than $50 \%$ of malpositioned blindly inserted nasogastric feeding tubes occur in patients with endotracheal tube or tracheostomy [3]. The malpositioned nasogastric feeding tubes are not included in risk management databases, therefore they may well be underreported. This may ex-plain, at least in part why the data on malpositioned nasogastric tubes in mechanically ventilated patients are limited. Recent data suggest that malpositioned blindly inserted feeding tubes may cause serious thoracic complications in mechanically ventilated patients. Rassias et al. reported that of 13 mechanically ventilated patients with malpositioned blindly inserted feeding tubes 36\% had serious complications [4]. Similarly, Mardestein et al. reported that of 57 mechanically ventilated with malpositioned nasogastric feeding tubes $38 \%$ had serious thoracic complications [5]. However, these reports may be difficult to interpret as they investigated interventions to reduce malpositions of nasogastric and promote a safer placement of nasogastric tubes in mechanically ventilated patients.

This review outline the thoracic complications associated with the blind placement of the nasogastric feeding tube for mechanically ventilated patients in intensive care, and the methods used to verify the position and promote safer gastric placement of the nasogastric tubes.

\section{MALPOSITIONED BLINDLY INSERTED NASOGASTRIC FEEDING TUBES}

\subsection{Definitions and Incidence}

The development of nasogastric feeding tube is attributed to John Hunter in the late 1700s but it was not until 1976 that Dobbie and Hoffmeister developed the narrow-bore soft polyvinylchloride enteral feeding tube [1]. 
These tubes reduced problems associated with the earlier large-bore tubes related to the patient's comfort and mucosal ischaemia due to compression by the tube. They usually have stylets which give stiffness and strength to the tubing and allow easier advancement of the device. However, not long after the introduction of narrow-bore feeding tubes into the clinical practice complications began to be reported. In 1978 James et al. described perforation of the oesophagus and mediastinum by a narrowbore non-proprietary nasogastric tube in a mechanically ventilated patient. He concluded that cardiomegaly had resulted in significant oesophageal distortion leading to perforation by the nasogastric tube [6].

A nasogastric feeding tube is considered malpositioned if it does not stay within the oesophageal lumen or if the distal end of the tube is not below the gastrooesophageal junction. To place a nasogastric tube we assume the median distance from the anterior nasal spine to the tracheooesophageal junction to be about $20 \mathrm{~cm}$, the oesophagus to be $25 \mathrm{~cm}$ long and aim for the tip of the nasogastric tube to lie $10 \mathrm{~cm}$ below the gastro-oesophageal junction. Therefore, ideally the nasogatsric tube should be secured at the 50 to $60 \mathrm{~cm}$ mark at the nasal vestibule [7]. Alternatively, adding the distance from the nose to the pinna and the distance from the pinna to the xiphoid process and adding another $5 \mathrm{~cm}$, will place the tip of the nasogatsric tube in the fundus of the stomach.

Mc Wey et al. reported an incidence of malpositioned tubes of $1.3 \%$ of which $61 \%$ occurred in mechanically ventilated patients [8]. Sorokin et al. reported an incidence of malpositioned tubes of $1.3 \%$ of which $>50 \%$ occurred in mechanically ventilated patients, with a procedure related rate of serious thoracic complications of
$28 \%$ [3]. Mardestein et al. reported malpositioned feeding tubes in $2 \%$, of which $67.8 \%$ occured in mechanically ventilated patients, with serious thoracic complications in 38\% [5]. Rassias et al. found malpositioned nasogastric feeding tubes in $2 \%$, of which $92 \%$ occurred in mechanically ventilated with serious complications in $35 \%$ [4]. Nascimento et al. reported an incidence of malpositioned tubes in a tertiary care referral hospital of $3 \%$ [1]. Although the figure of three malpositioned tubes in every 100 patients that had this procedure may not seem particularly high at first glance, the fact that $40 \%$ of the malpositioned tubes led to pneumothorax and 4 patients died raised the authors concern. They translated this figure into one death every three months in a tertiary care hospital from a "simple" procedure.

\subsection{Thoracic Complications in Mechanically Ventilated Patients}

Malpositioned blindly inserted nasogastric feeding tubes were divided by Pillai et al. [7] into tubes which were malpositioned and remained intrathoracic and tubes which were malpositioned but did not remain intrathoracic, the majority of which were misplaced intracranially. Eighty three patients who were mechanically ventilated in intensive care were reported in the literature to have an intrathoracic malpositioned blindly inserted nasogastric feeding tube. Of them 55 (66\%) mechanically ventilated patients had thoracic complications from the malpositioned nasogastric tubes that required treatment other than observation as it is now presented in Table 1 [4,6-32]. Some patients had more than one thoracic complications since 59 complications were described in 55 mechanically ventilated patients in intensive care (Table 1).

Table 1. Summary of thoracic complications from malpositioned nasogastric feeding tubes in mechanically ventilated patients in intensive care.

\begin{tabular}{lcc}
\hline & N & Reference \\
\hline Patients with thoracic complications that required treatment & 55 & $4,6-32$ \\
Complications that required treatment & 59 & $4,6-32$ \\
Pneumothoraces that required ICD & 44 & $4,6,8-11,13,16,17,21,23-31$ \\
Right & 20 & $8-11,13-16,21,23-25,28,29$ \\
Left & 3 & $8,10,14$, \\
Bilateral & 2 & 26 \\
Side not stated & 19 & $4,17,27,30,31$ \\
Pneumonias treated with antibiotics & 9 & $8,9,14,17,18,20$ \\
Knotting of NGT that needed sedation to remove it and steroids & 3 & $19,22,23,32$, \\
Oesophageal perforation-mediastinitis treated with antibiotics & 2 & 6,8 \\
Loss of tidal volume that required manual ventilation & 1 & 12 \\
\hline
\end{tabular}

Abbreviations: NGT, nasogastric tube; ICD, Intercostal drain. 
Ninety one percent of the reported thoracic complications were tracheobronchopleural (Table 1). The commonest tracheobronchial complication was pneumothorax, which occurred more commonly on the right side and accounted for $80 \%$ of complications. It was treated with intercostal drains which were kept in place from a few days up to 2 weeks. Pneumonia occurred in $16 \%$. Others have reported higher rates of pneumonia following a malpositioned tube. Bankier et al. reported that $40 \%$ of 10 patients developed pneumonia at the former site of the tip of the malpositioned tube [9]. None of them had evidence of pleural perforation or alimentary feeding over the malpositioned tube. This difference between the rate of pneumonia from malpositioned nasogastric tubes between the data of Bankier et al. and those of Malderstein et al. may be explained at least in part by the differrent methods used to diagnose pneumonia in mechanically ventilated patients $[5,9]$. Knotting of nasogastric feeding tube around the epiglottis or around the endotracheal tube has been reported to cause vocal cords oedema that was treated with removal of the nasogastric tube under direct laryngoscopy and hydrocortisone [19]. Loss of tidal volume was described when an intrabrochially misplaced nasogastric feeding tube produced, through its connection with negative pressure suction, constant flow of gas out of the airways and rapid ventilator triggering [12] The reported incidence of oesophageal perforation from malpositioned nasogastric feeding tubes appear to be low in mechanically ventilated patients $[6,8]$. The introduction of soft narrow bore feeding tubes may have some contribution to the low incidence of oesophageal perforation [10]. This complication may be more common when intubated patients with mediastinitis have surgical manipulation or repetitive advancement of the nasogastric tube under general anaesthesia [33].

Large volume low pressure cuffs are now in use in intensive care since they cause less mucosal compression and necrosis and reduce some of the complications associated with long term intubation. However, because the large volume low pressure cuffs are softer than the low volume high pressure cuffs and remain wrinkled even when inflated and occluding the tracheal lumen, a wireguided narrow-bore nasogastric feeding tube may be introduced into the trachea. Moreover, endotracheal misplacement of a narrow-bore nasogastric feeding tube can be facilitated by endotracheal tubes that are large compared to the tracheal lumen or have a small cuff diameter to tracheal diameter ratio. Misplacement of large bore feeding tubes without stylet into the trachea of mechanically ventilated non-sedated patients with tracheostomy tubes of inner diameter $8.5 \mathrm{~mm}$ has also been reported $[18,19]$. Under these circumstances the endotracheal tube did not appear to act as a barrier to the passage of a large or narrow bore feeding tube with or without stylet down the trachea. However, sedation and obtundation very often accompany ventilator support. Thus, the endotracheal or tracheostomy tube may simply be a marker for altered or unstable mental status which in turn may be the true risk factor that depresses the airway reflexes. Of the 55 mechanically ventilated patients with malpositioned nasogastric tubes $42(76.36 \%)$ were sedated or not alert.

The episodes of endotracheal misplacement of a narrow bore nasogastric tube were evenly distributed between tubes with and without stylet (Table 2). While stylet migration may be a concern in cases of pulmonary perforation, we know no case of a bronchial perforation by a metal stylet that slipped out of the nasogastric tube in mechanically ventilated patient. While metal stylets may be regarded as facilitating pneumothoraces by stiffening the tube, insertion of a nasogastric tube may be much easier with the stylet in place.

Woodall et al. initially suggested that narrow tipped tubes perforated the lungs more easily than bulbous tipped tubes, in their series of 17 patients insertion into the distal bronchial tree led to pneumothorax regardless of the tube type whereas insertion into the proximal bronchial tree did not [34]. The blind element of insertion and the distance at which the tube is advanced appear to have more significant contribution to misplacement and the relevant complications than the type of the nasogastric tube.

\subsection{Diagnosis of Malpositioned Feeding Tubes}

The diagnosis of malpositioned nasogastric tube insertion in most of the cases reported was made by the post procedural radiograph, which in several cases did not occur immediately. However, there are reports where the diagnosis occurred during the procedure, immediately afterwards or even post hospital discharge. A 67 year old patient was complaining of epigastric pain of 3 months duration two years after being discharged from intensive care following a triple vessel coronary artery bypass graft. Endoscopy reported a gastric ulcer associated with a tubular foreign body which was proved to be the endotracheal tube that was used to facilitate the insertion of the nasogastric tube [35].

The majority of malpositioned nasogastric feeding tubes have uneventful insertion without feeling of resistance or malposition. Only in $9 \%$ of the 55 cases with malpositioned nasogastric feeding tubes it was reported that the operator felt sensation of resistance that alerted him or her of the possibility of misplacement.

Radiographic and non-radiographic methods to confirm the placement of nasogastric tubes are in use. The nonradiographic indicators of tube placement are aspiration 
Table 2. Characteristics of the malpositioned nasogastric feeding tubes that were used in 55 mechanically ventilated patients with thoracic complications.

\begin{tabular}{|c|c|c|}
\hline & $\mathrm{N}$ & References \\
\hline Malpositioned nasogastric tubes associated with thoracic complications & 55 & $4,6-32$ \\
\hline \multicolumn{3}{|l|}{ Time from tube insertion to diagnosis } \\
\hline 1. $<12 \mathrm{~h}$ & 13 & $4,11,14,10,12,21-25,32$ \\
\hline 2. $12-24 \mathrm{~h}$ & 6 & $4,18,28$ \\
\hline 3. Not stated & 36 & $6,8,9,13-17,26,27,29-31$ \\
\hline \multicolumn{3}{|l|}{ Nasogastric tubes } \\
\hline 1. with stylet & 20 & $8,10,11,13,14,16,17,21,25$ \\
\hline 2. without stylet & 17 & $4,9,18,22,26$ \\
\hline 3. Not stated & 18 & $6,11,12,15,19,20,23,24,27-32$ \\
\hline \multicolumn{3}{|l|}{ Tube position verified by } \\
\hline 1. X-ray only & 37 & $4,8,9,12,15-17,20,21-24,27,30$ \\
\hline 2. Auscultation or aspiration & 7 & $17,18,26,28,29,31$ \\
\hline 3. X-ray post auscultation or aspiration & 7 & $6,10,11,13,14,25$ \\
\hline 4. Laryngoscopy & 4 & $19,22,23,32$ \\
\hline \multicolumn{3}{|l|}{ Tube insertion } \\
\hline 1. Easy without resistance & 25 & $4,6,8,10-12,14-16,18,19,21-25$ \\
\hline 2. Difficult & 5 & $4,23,28,32$ \\
\hline 3. Not stated & 25 & $9,13,17,20,26,27,29-31$ \\
\hline Pneumothoraces on removal of a malpositioned NGT & 5 & $21,23,24,29$ \\
\hline Recurrent pneumothoraces & 5 & $9,10,17,26,27$ \\
\hline \multicolumn{3}{|l|}{ Patient outcome } \\
\hline 1. Discharge & 22 & $4,8,11,15-18,23,27-29$ \\
\hline 2. Death & 10 & $4,6,10,14,17,19,21,27$ \\
\hline 3. Not stated & 23 & $9,12,13,17,20,22,24-26,30-32$ \\
\hline
\end{tabular}

of gastric fluid, measurement of the gastric fluid ph and auscultation over the epigastrium of air injected through the nasogastric tube. The reported sensitivity of aspiration and measurement of gastric fluid ph was respectively $77 \%$ and $49 \%$ whereas the reported specificity was $38 \%$ and $74 \%$ [36]. The authors suggested that the combination of the three non-radiographic indicators did not improve the sensitivity and specificity. Aspiration of fluid should not be interpreted as appropriate placement in the stomach because fluid aspirated may come from the pleural space, bronchial secretions, stomach or even brain [37]. There are several reasons why insufflation of air with sounds heard over the left upper quadrant should not be accepted as evidence of gastric tube placement: 1) small bore tubes do not always allow sufficient passage of air; 2) vigorous peristalsis may be mistaken for insufflated air; 3) air bubbling in the pleura, lung, pharynx or oesophagus may be transmitted below the diaphragm [38].

X-ray remains the most accurate although not infallible method to verify proper nasogastric feeding tube placement [39]. Hendry et al. reported that of 11 patients with malpositioned nasogastric tubes, all of which had radiography for tube placement confirmation, the malposition was not identified in $27 \%$ [40]. The non-identified malpositioned tubes in that report had been placed in the left costophrenic sulcus which is difficult to distinguish from intragastric placement. The practice alert issued in 2005 by the American Association of Critical Care Nurses recommends radiographic confirmation of correct tube placement prior to initial use in all critically ill patients receiving feedings or medications via blindly inserted tubes. Although not explicitly stated this recommendation refers to all blindly inserted tubes regardless of size [41]. Within this context many institutions now require X-ray confirmation not only of the position of the tube in the gastrointestinal tract but also that the ports of a nasogastric tube are in the stomach and therefore it is properly positioned in the gastrointestinal tract. There are reports of patients who aspirated through nasogastric tubes that ended in the distal oesophagus. Many experts and professional bodies agree that the cost of an X-ray to confirm correct placement of a blindly inserted tube of any size prior to its use to administer formula or medications should be regarded as a justified expense [42]. 
X-ray may correctly identify the majority of misplaced nasogastric feeding tubes and prevent intrapleural or intrabronchial alimentation. However, it does not appear to prevent pneumothoraces and consequently does not spare the patients from subsequent intercostal drains and prolonged stay in health care facilities.

\subsection{Methods to Increase the Safety of Blind Insertion of Feeding Tubes}

Different methods to increase the safety of blind insertion of nasogastric tubes have been developed. Direct visualization of the larynx with laryngoscopy and posterior passage of the tube has been used with positive results $[43,44]$. Fluoroscopy has been another option as the tubes are radioopaque. Roubennof et al. calculated that fluoroscopy involve $0.01 \mathrm{~Gy} / \mathrm{min}$ which for a procedure of 5 mins involves $50 \mathrm{mGy}$ per fluoroscopic insertion [45]. Ultrathin transnasal endoscopy has also been tested in patients with oesophageal cancer and was successful in $99 \%$ of cases [46]. However, fluoroscopy and endoscopy may require equipment that is not always available or transfer of a critically ill to the radiology or endoscopy department.

Thomas et al. used a colorimetric end-tidal $\mathrm{CO}_{2}$ indicator device, that has been attached to the proximal end of a small bore feeding tube to test, in 11 mechanically ventilated patients, whether it would reliably discriminate between those tubes passed into the airways and those passed into the alimentary tract [47]. The device uses a sulphapthalein impregnated ph-sensitive filter paper which changes from purple to yellow in the presence of $\mathrm{CO}_{2}$. The authors suggested that it was $100 \%$ sensitive and specific and that the cost compared favourably to radiographic evaluation (14.50 USD for the capnograph detector and 60.45 for a single portable chest radiograph, exclusive of nursing and physician's time). This device has also been tested by another group in a two-phase study in mechanically ventilated patients [48]. First, the authors reported $100 \%$ accuracy in 10 intubated patients in whom the feeding tube was deliberately placed into the endotracheal tube. Then they retested the method in 93 intubated patients in whom the tube was inserted in a two-step radiological technique. After advancing the tube approximately $25 \mathrm{~cm}$ from the lips, insertion was stopped and the colorimetric determination of placement was made before a radiograph was taken. The method correctly identified seven of eight tracheal placements with a sensitivity of $88 \%$ and specificity of $99 \%$. However, with insertion of gastric tubes, especially in recumbent critically ill patients, gastric contents may flow back up the tube and moisten the colorimetric indicator. In these instances, clinicians should proceed with other verification methods rather than rely on the colorimetric indicator. Although promising, this technology is not widely available. Therefore it may not be a replacement for radiography but a method of improving safety by preventing potential airway or lung intubation during placement of a gastric tube.

A two-step insertion of a nasogastric feeding tube has been tested by Mardestein et al. [5]. These authors applied a protocol which required a pause when the nasogastric tube has reached $35 \mathrm{~cm}$, so that a radiograph could confirm that the tube had not been placed in a bronchus. If the tube was determined to be in the oesophagus, it could be safely advanced without danger of passing into the distal tracheobronchial tree. A second chest X-ray was performed when the nasogastric tube was put in place to verify the position of the tube in the stomach. Although, this approach has been initially proposed by Roubenoff et al., it has not been tested until Mardestein et al. reported that with the two step approach no tube placed in the oesophagus has caused pulmonary damage $[5,45]$. The post-intervention incidence of pneumothorax among patients having an intrabronchial feeding tube has been reduced to $3.3 \%$ from the pre-intervention rate of $26.9 \%$. The contrary view was that the extra cost of an additional X-ray for each patient with a nasogastric tube may not be affordable on a wider scale [49]. The argument was that we don't have the data that would justify system wide interventions.

Sorokin et al. applied a policy to reduce pneumothoraces from nasogastric feeding tubes in intubated or sedated patients [3]. They applied three changes: 1) They initially limited who inserted nasogastric tubes to intubated or sedated patients and then evaluated the benefit of using a safer tube and a safer two-step method of insertion. The tubes were either not advanced beyond $35 \mathrm{~cm}$ until a radiograph was obtained or were advanced under direct laryngoscopic, fluoroscopic or capnometric guidance. 2) The feeding tubes malpositions and related complications were monitored and reported regularly to the clinical staff and 3) resident education program on feeding tube insertion was started. The contrary view was that smaller institutions may not afford a specialized team devoted to feeding tube placement.

Haddad et al. used an external transmitter with an electromagnetic pulse sensor system at the bedside to observe the location of the tip of the tube as it is manually inserted [50]. Although not thoroughly studied preliminary reports suggest high success rates and decreased insertion time. Bercik et al. studied a magnet - tipped computer tracking system and compared it with simultaneous fluoroscopic and manometric monitoring [51]. The authors reported excellent correlation between the three techniques.

Our review has limitations. At first it is subject to the 
problems inherent to retrospective data collection. Second we may underestimate the rate of malpositions and complications. This is so because clinicians or nursing staff may not report all the malpositioned tubes that may occur in the same patient, since this procedure does not require informed consent and in several countries including USA does not have a billing record. Additionally patients experiencing a pneumothorax after a difficult tube placement may have been missed if the tube had been removed before obtaining the X-ray. Third we included specialized and general intensive care units.

\section{CONCLUSIONS}

The insertion of a malpositioned nasogastric feeding tube is not included in risk management databases, does not require informed consent and may well be underreported. However, it may cause serious complications in mechanically ventilated patients that may extend the patients stay in expensive intensive care facilities. More than $50 \%$ of mechanically ventilated patients with reported malpositioned nasogastric feeding tubes had serious thoracic complications. Several approaches to reduce the rate of complications have been proposed. Although a zero complication rate is desirable the data available does not suggest that it is affordable by smaller institutions that are unable to devote the resources, either personnel or money of a specialized team or a second X-ray for every nasogastric feeding tube or up to date technology. Data on the complications from blindly inserted nasogastric feeding tubes in mechanically ventilated patients are urgently needed, to measure the accurate burden of thoracic complications from malpositioned nasogastric feeding tubes. Then we would be able to answer the question of whether we can afford to prevent them. Until then direct laryngoscopy to verify that the tube is in the oesophagus, followed by X-ray as soon as possible to confirm that all ports are in the stomach and certainly before any use of the tube seems to be a reasonable and affordable approach, that many clinicians apply for sedated mechanically ventilated patients in intensive care.

\section{REFERENCES}

[1] De Aguilar-Nascimento, J.E. and Kudzu, K.A. (2007) Clinical costs of feeding tube placement. Journal of Parenteral and Enteral Nutrition, 31(4), 269-273.

[2] National Patient Safety Agency (2005) Reducing the harm caused by misplaced nasogastric and orogastric feeding tubes in babies under the care of neonatal units. http//www.npsa.nhs.uk/site/media/documents/1296_Patie ntsSafety Alert.pdf

[3] Sorokin, R. and Gottlieb, J. (2006) Enhancing patient safety during feeding tube insertion: A review of more than 2000 insertions. Journal of Parenteral and Enteral
Nutrition, 30(5), 440-445.

[4] Rassias, A.J., Ball, P.A. and Corwin, H.L. (1998) A prospective study of tracheopulmonary complications associated with the placement of narrow bore enteral feeding tubes. Critical Care, 2(1), 25-28.

[5] Mardestein, E.I., Simmons, R.L. and Ochoa, J.B. (2004) Patient safety: Effect of institutional protocols on adverse events related to feeding tube placement in the critically ill. Journal of the American College of Surgeons, 199(1), 41-47.

[6] James, R.H. (1978) An unusual complication passing a narrow bore nasogastric tube. Anaesthesia, 33(8), 716718.

[7] Pillai, J.B., Vegas, A. and Brister, S. (2005) Thoracic complications of nasogastric tube; review of safe practice. Interactive Cardiovascular and Thoracic Surgery, 4(5), 429-433.

[8] Mc-Wey, R.E., Curry, N.S., Schabel, S.I. and Reines, D. (1988) Complications of nasoenteric feeding tubes. American Journal of Surgery, 155(2), 253-257.

[9] Bankier, A.A., Wiesmayr, M.N., Turetscheck, K., Mallek, R., Fleischmann, D., Janata, K. and Herold, C.J. (1997) Radiographic detection of intrabronchial malpositions of nasogastric tubes and subsequent complications in intensive care unit. Intensive Care Medicine, 23(4), 406-410.

[10] Theodore, A.C., Frank, J.A., Ende, J., Snider, G.L. and Beer, D.J. (1984) Errant placement of nasoenteric tubes. A hazard in obtunded. Chest, 86(6), 931-933.

[11] Kawati, R. and Rubertson, S. (2005) Malpositioning of fine bore feeding tube: A serious complication. Acta Anaesthsiol Scan, 49(1), 58-61.

[12] Tenholder, M., Erwin, W. and Nelson, H. (1994) Lost tidal volume in a 71-year old ventilated patient. Chest, 106(6), 1869-1871.

[13] Ibara-Perez, C. (1992) Perforación pulmonar por sonda de pequeño calibre para alimentación enteral. Revista de Invvestigation Clinica, 44(2), 255-258.

[14] Druml, W., Gleinberg, G., Base, W., Haller, J., Laggner, A. and Lenz, K. (1984) Lung perforation by nasogastric feeding tubes. Clinical Nutrition, 2(3), 197-199.

[15] Hand, R., Kempster, M. and Levy, J. (1984) Inadvertent transbronchial insertion of narrow-bore feeding tube sinto the pleural space. Journal of the American Medical Association, 251(18), 2396-2397.

[16] Eldar, S. and Meguid, M. (1984) Pneumothorax following attempted nasogastric intubation for nutritional support. Journal of Parenteral and Enteral Nutrition, 8(4), 450-451.

[17] Valentine, J. and Turner, W. (1985) Pleural complications with nasoenteric feeding tubes. Journal of Parenteral and Enteral Nutrition, 9(5), 605-607.

[18] Wang, P.C., Tseng, G.Y., Yang, H.B., Chou, K.C. and Chen, C.H. (2008) Inadvertent tracheobronchial placement of feeding tube in a mechanically ventilated patient. Journal of the Chinese Medical Association, 71(7), 365367.

[19] Agarwal, A., Gaur, A., Sahu, D., Singh, P. and Pandey, C. (2002) Nasogastric tube knotting over the epiglottis: A cause of respiratory distress. Anesthesia \& Analgesia, 94(6), 1659-1660.

[20] Stark, P. (1982) Inadvertent nasogastric tube insertion into the tracheobronchial tree. Radiology, 142(1), 239- 
240.

[21] Balogh, G.J., Adler, S.J., Vander Woude, J., Glazer, H.S., Roper, C. and Weyman P.J. (1983) Pneumothorax as a complication of feeding tube placement. American Journal of Roentgenology, 141(6), 1275-1277.

[22] Trujillo, M.H., Fragachan, C.F., Tordoledo, F. and Ceballos, F. (2006) Lariat loop knotting of a nasogastric tube: An ounce of prevention. American Journal of Critical Care, 15(4), 413-414.

[23] Biggart, M., McQuillan, P., Choudrhry, A.K. and Nickalis, R.W. (1987) Dangers of placement of narrow bore nasogastric feeding tubes. Annals of the Royal College of Surgeons of England, 69(3), 119-120.

[24] Weinberg, L. and Skewes, D. (2006) Pneumothorax from intrapleural placement of a nasogatsric tube. Anaesth Intensive Care, 34(2), 276-279.

[25] Kolbitsch, C., Pomaroli, A., Lorenz, L., Gassner, M. and Luger, T.J. (1997) Pneumothorax following nasogastric feeding tube insertion in a tracheostomized patient after bilateral lung transplantation. Intensive Care Medicine, 23(4), 440-442.

[26] Miller, K.S., Tomlinson, J.R. and Sahn, S.A (1985) Pleuropulmonary complications of enteral tube feedings. Two reports, review of the literature and recommendations. Chest, 88(2), 230-233.

[27] Scholten, D.J., Wood, T.L. and Thompson, D.R. (1986) Pneumothorax from nasoeneteric feeding tube insertion. A report of five cases. American Journal of Surgery, 52(7), 381-385.

[28] Hensel, M. and Martniz, R. (2010) Pneumothorax following nasogastric feeding tube insertion. Case report and review of the literature. Anaesthesist, 31(2), (Epub Ahead of Print).

[29] Idu, S.R. and van de Leur, J.J. (1998) Iatrogenic tension pneumothorax following faulty positioning of gastric tube. Nedderlands Tijdschrift voor Geneeskunde, 142(3), 142-145.

[30] Scheffenr, S.E., Gross, B.H., Birnberg, F.A. and Birk, P. (1985) Iatrogenic bronchopleural fistula caused by feeding tube insertion. Journal of Canadian Association Radiology, 36(1), 52-55.

[31] Nakao, M., Killam, D. and Wilson, R. (1983) Pneumothorax secondary to inadvertent nasotracheal placement of a nasoenteric tube past a cuffed endotracheal tube. Critical Care Medicine, 11(3), 210-211.

[32] Bautiista, E. (1988) Complications of nasogastric feeding tube insertion. Chest, 93(5), 1119-1120.

[33] Wu, P.Y., Kang, T.J., Hui, C.K., Sun, W.Z. and Chan, W.H. (2006) Fatal massive hemorrhage caused by nasogastric tube misplacement in a patient with mediastinitis. Journal of the Formosan Medical Association, 105(1), 80-85.

[34] Woodall, B.H., Winfield, D.F. and Bisset, G.S. (1987) Inadvertent tracheobronchial placement of feeding tubes. Radiology, 165(3), 727-729.

[35] Rousseau, P., Mulanu, C. and Aunac, S. (2006) Gastric foreign body. Rare complication of nasogastric tube placing. Annales Françaises d'Anesthèsie et de Rèanimation, 25(7), 777-779.

[36] Seguin, P., le Bouquin, V., Aguillon, D., Maurice, A.,
Lavioll, B. and Malledant, Y. (2005) Testing nasogastric tube placement: evaluation of three different methods in intensive care. Annales Françaises d'Anesthèsie et de Rèanimation, 24(6), 594-599.

[37] Metheny, A., Meert, K.L. and Clouse, R.E. (2007) Complications related to feeding tube placement. Current Opinion in Gastroenterology, 23(2), 178-182.

[38] Wendel, G.D., Lenchner, G.S. and Promisloff, R.A. (1991) Pneumothorax complicating small-bore feeding tube placement. Archives of Internal Medicine, 151(3), 599-602.

[39] Metheny, N.A. (2006) Acute complications associated with bedside placement of feeding tubes. American Journal of Critical Care, 21(1), 40-55.

[40] Hendry, P.J., Akyurekli, Y., McIntyre, R., Quarrington, A. and Keon, W.J. (1986) Bronchopleural complications of nasogastric feeding tubes. Critical Care Medicine, 14(10), 892-894.

[41] American Association of Critical Care Nurses Practice Alert (2005) Verification of feeding tube placement.

[42] Metheny, N. (2007) Confirmation of nasogastric tube placement. American Journal of Critical Care, 16(1), 19.

[43] Gombart, S., Khanna, A.K. and Gombar, K.K. (2007) Insertion of a nasogastric tube under direct vision: Another atraumatic approach to an old issue. Acta Anaesthesiol Scandinav, 51(7), 962-963.

[44] Jones, A.P., Diddee, R. and Bonner, S. (2006) Insertion of a nasogastric tube under direct vision. Anaesthesia, 61(3), 295-306.

[45] Roubenoff, R. and Ravich, W. (1989) Pneumothorax due to nasogastric feeding tubes. Archives of Internal Medicine, 149(2), 184-186.

[46] Lin, C.H., Liu, N.J., Lee, C.S., Tang, J.H., Wei, K.L., Yang, C., Sung K.F., Cheng, C.L., Chiu, C.T. and Chen, P.C. (2006) Nasogastric feeding tube placement in patients with oesophageal cancer: Application of ultrathin transnasal endoscopy. Gastrointest Endosc, 64(1), 104107.

[47] Thomas, B.W. and Falcone, R.E. (1998) Confirmation of nasogastric tube placement by colorimetric indicator detection of carbon dioxide: a preliminary report. Journal of the American College of Nutrition, 17(2), 195-197.

[48] Burns, S.M., Carpenter, R., Blevins, C., Bragg, S., Marshall, M. and Browne, L. (2006) Detection of inadvertent airway intubation during gastric tube insertion: Capnometry versus a colorimetric carbon dioxide detector. American Journal of Critical Care, 15(2), 188-195.

[49] Reid, A. (2004) Feeding tube placement in the critically ill. Invited commentary. Journal of the American College of Surgeons, 199(1), 47-48.

[50] Haddad, N., Nawaz, T., Potter, L. and Lipman, T. (1993) Avoidance of nasopulmonary intubation by feeding tubes with stethoscopic guidance: "The stethotube". Gastroenterology, 104(5), A623.

[51] Bercik, P., Schlageter, V., Mauro, M., Rawlinson, J., Kucera, P. and Armstrong, D. (2005) Non-invasive verification of nasogastric tube placement using a magnet tracking system: A pilot study in healthy subjects. Journal of Parenteral and Enteral Nutrition, 29(4), 305-310. 